\title{
ZUR WIRTSCHAFTSGEOGRAPHISCHEN SITUATION DER FÄRÖER
}

\author{
Gerhard Oberbeck
}

\begin{abstract}
Die folgenden Darlegungen gelten den Färöern, die - obwohl zu Dänemark gehörend ein ganz eigenes und von mitteleuropäischen Verhältnissen sehr abweichendes Bild aufweisen. Die isolierte geographische Lage, die wenig günstigen natürlichen Gegebenheiten und die historische Entwicklung haben ein in manchen Zügen recht selbständiges kleines «Land» entstehen lassen, dessen Bewohner auch durchaus den Anspruch auf eine völkische Eigenständigkeit erheben, eine Tatsache, die der dänische Staat in Form von färischen Vorrechten (z.B. eigenem «Lagting») zu berücksichtigen versucht hat. So ist es berechtigt, über die wirtschaftsgeographische Situation der Inseln gesondert zu berichten, zumal diese in der deutschen und schweizerischen Literatur bisher wenig Beachtung gefunden haben 1.
\end{abstract}

Die Gruppe der Färöer oder Schafsinseln (fär. Föroyar, dän. Færøerne) besteht aus 18 einzelnen Eilanden, von denen 17 bewohnt sind. Sie liegt etwa zwischen $62^{\circ} 24^{\prime}$ und $61^{\circ} 24^{\prime}$ nördlicher Breite und $6^{\circ} 15^{\prime}$ und $7^{\circ} 41^{\prime}$ westlicher Länge, d.h. ein wenig südlicher als die Südspitze Islands, etwas nördlicher als der südlichste Teil Grönlands und auf etwa der gleichen nördlichen Breite wie die am weitesten nach Westen reichende Spitze der skandinavischen Halbinsel. Bei einer Nord-Süd-Erstreckung von ungefähr $113 \mathrm{~km}$ und einer West-Ost-Ausdehnung von etwa $75 \mathrm{~km}$ besitzen die Färöer eine Fläche von $1399 \mathrm{~km}^{2}$.

Die gebirgige Oberfläche ist für die Anlage von menschlichen Siedlungen recht ungünstig. Auf engstem Raum steigt das Niveau bis auf $862 \mathrm{~m}$ (Slættaratindur auf Eysturoy) an, unterbrochen von zahlreichen, tief eingeschnittenen Fjorden mit vielfach nahezu senkrechten Wänden. Die Westküsten, fast ausnahmslos als Steilküsten mit Höhen von $300-400 \mathrm{~m}$ und vereinzelt bis $750 \mathrm{~m}$ ausgebildet, weisen nur an ganz wenigen Stellen kleinere Buchten auf; dementsprechend finden sich die meisten Orte an den flacheren, durch zahlreiche Fjorde gegliederten Ostseiten der Inseln.

Das morphologische Bild ist in starkem Maße bedingt durch die geologische Beschaffenheit des Untergrundes. Basalt- und Tuffdecken, oft zwanzig- und dreißigfach übereinandergelagert, kennzeichnen den relativ einfachen geologischen Aufbau, der mit zahlreichen Eruptionsphasen während des Tertiärs erklärt wird (u.a. RudolPHI, 1913, S. 162; BøGGILD, 1928, S. 188). Im Miozän erfolgte der Abschluß dieser Entwicklung. Die anschließende Periode der Senkungen und Einbrüche hat dann in Verbindung mit Denudation und Erosion die heutige fjord- und buchtenreiche Inselwelt entstehen lassen, wobei der unterschiedliche Härtegrad der Basalt- und Tuffschichten sich auf die Oberflächengestalt entscheidend ausgewirkt hat; da die Tuffe wesentlich stärker der Verwitterung anheimfallen, konnte sich eine treppenartige Abstufung der Schichten herausbilden, die bis zur Gegenwart ein beträchtliches Hindernis sowohl für die wirtschaftliche Nutzung des Landes als auch für seine verkehrsmäßige Erschließung gewesen ist (vgl. Abb. 1). Infolge eines geringen Einfallens der Gesteinsschichten nach Ost oder Südost dacht die Oberfläche leicht nach Osten ab und gewinnt stellenweise den Charakter von kleineren Plateaus, der Küstensaum bleibt aber dennoch fast ausnahmslos recht schmal.

Auf die heutige Oberflächengestalt hat sich ferner in starkem $\mathrm{Maße}$ eine pleistozäne Vereisung ausgewirkt, als deren Zeugen neben zahlreichen Geschieben Gletscherschrammen, Rundhöcker, vereinzelte Seiten- und Grundmoränen und vor allem die

1 Die Ausführungen gründen sich auf Beobachtungen und Archivstudien des Verfassers während zweier längerer Aufenthalte auf den Inseln (Sommer 1956 und Sommer 1957). Der Deutschen Forschungsgemeinschaft, die den Abschluß der Untersuchungen ermöglichte, sei dafür der verbindlichste Dank gesagt. 
Abb. 1: Funningsfjord auf Eysturoy mit dem $697 \mathrm{~m}$ hohen Húsáfelli. Das am Fjordende (Vordergrund) gelegene kleine Dorf Funningsbótnur ist durch keine Straße mit anderen Orten verbunden.

(Photo:

OBERBECK 57|1244)

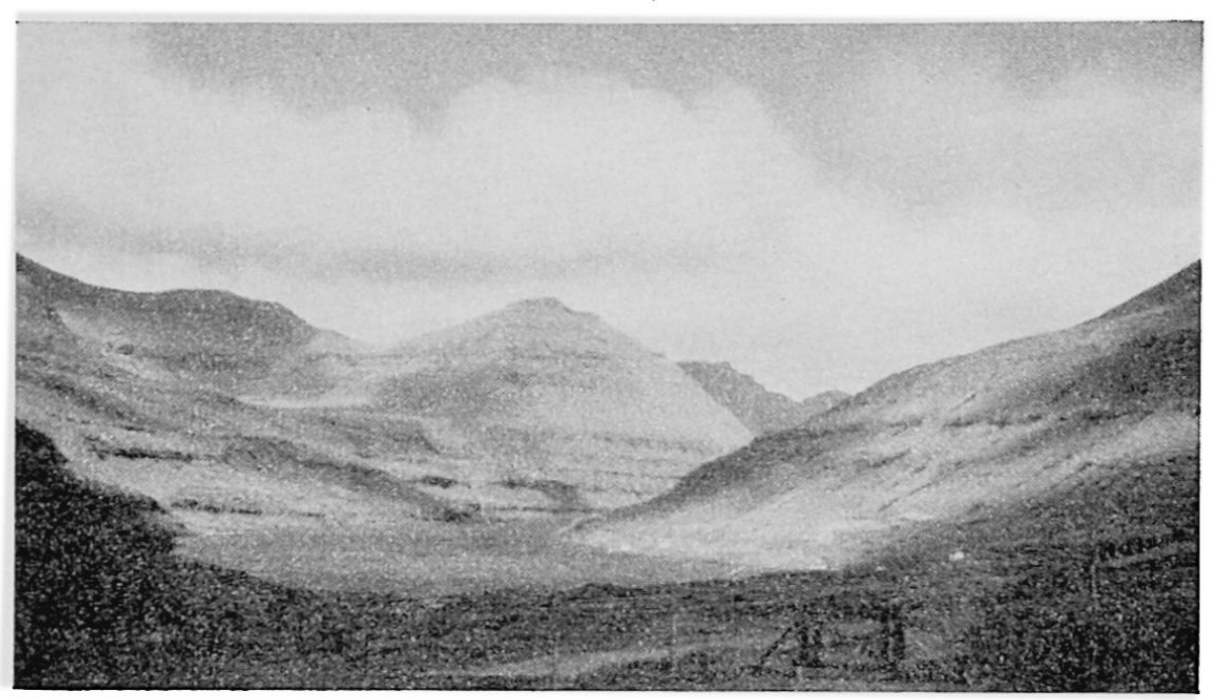

überwiegend auf den gebirgigeren nördlichen Inseln vorhandenen Großkare, deren Wände Höhen bis zu $400 \mathrm{~m}$ aufweisen, erwähnt seien. Hinzu kommen eine große Anzahl von glazialen Seen (nahezu 800), die zum Teil als Rinnen- und Schmelzwasserseen, zum Teil aber auch als Karseen ausgebildet sind, sowie einige Strandseen aus jüngerer Zeit.

Das Klima der Färöer ist trotz der hohen Breitenlage relativ mild; es zeichnet sich durch beträchtliche Niederschläge, ausgeglichene Temperaturen und recht kräftige, wechselnde Winde aus, die während des Winterhalbjahres sehr oft den Charakter von Stürmen annehmen. Gemäß dem ozeanischen Klima weisen die Temperaturen innerhalb eines Tages und innerhalb eines Jahres nur unwesentliche Unterschiede ${ }^{2}$ auf, und auch Niederschläge sind für alle Jahreszeiten ${ }^{3}$ typisch.

Die Vegetation entspricht den ungünstigen klimatischen und morphologischen Verhältnissen der Inseln. Eine nur dünne Humusdecke, die bis zu einer Höhenlage von $350 \mathrm{~m}$, ganz selten bis zu $500 \mathrm{~m}$ anzutreffen ist, trägt einen zum Teil recht kräftigen und für Schaf- und Rinderzucht gut geeigneten Grasbewuchs. Dagegen fehlen Bäume und Sträucher fast völlig, was einerseits durch die heftigen Winde und die geringe Sommerwärme, zum anderen aber auch durch den Schafverbiß zu erklären ist.

Abgesehen von zwei kleineren Ortschaften liegen die anderen 119 Siedlungen $^{4}$ der Inseln in der meist nur schmalen Küstenzone einer Bucht oder eines Fjordes, wobei die 6 sehr gebirgigen Nordinseln insgesamt lediglich 21 - mit Ausnahme von Klakksvik - überwiegend kleine Orte aufweisen (vgl. Abb.3). Dagegen sind die beiden Hauptinseln Eysturoy und Streymoy mit 37 bzw. 26 sowie die südliche Insel Suðuroy mit 15 Städten und Dörfern am stärksten besiedelt.

Ein ähnliches Bild ergibt sich für die Bevölkerungsverteilung. Nach der letzten amtlichen Einwohnerstatistik (1955) lebten 15\% der insgesamt knapp 32400 Menschen 5 im Nordinselbezirk, 30\% auf Streymoy, 22\% auf Eysturoy, 19\% auf Suðuroy und der Rest auf den übrigen Inseln. Die größten Orte, Tórshavn auf Streymoy, Klakksvik auf Borðoy und Tvöroyri-Trongisvágur auf Suðuroy, zählten 1955 ca. 6100, 3300 bzw. 1700 Einwohner.

2 In Tórshavn beträgt die Durchschnittstemperatur für den Juli (wärmster Monat) $10,8^{\circ}$, für den Februar (kältester Monat) $3,1^{\circ}$ und für das Jahr 6,5 (vgl. Angaben in den einzelnen Bänden des Statistisk $\AA$ rbog).

3 In Tórshavn fallen im Durchschnitt $1500 \mathrm{~mm}$ Niederschläge pro Jahr; die Anzahl der Regentage beläuft sich auf jährlich 280 .

4 Statistisk Årbog, 1957, S. 312.

5 Ende 1955 betrug die Gesamtbevölkerung 32380 Personen (The Statesman's Year-Book, 1958, S. 942). 
Die Haupternährungs- und Einnahmequelle für die Färinger ist der Fischfang, der sowohl in Form der Küsten- als auch der Hochseefischerei betrieben wird. War man ursprünglich nur daran interessiert, den eigenen Bedarf zu decken, so ging man nach der Einführung des Freihandels und der Auflösung des dänischen Handelsmonopols (1856) in immer stärkerem Maße dazu über, auch zu exportieren. $\mathrm{Zu}$ Beginn des 20.Jahrhunderts umfaßte die färische Flotte bereits 129 Fischereischiffe (1906) mit insgesamt $10037 \mathrm{t}^{6}$, d.h. etwa die achtfache Menge dessen, was 1885 an Schiffsraum vorhanden gewesen war. 1939 verfügten die Färöer über $158 \mathrm{Fahrzeuge} \mathrm{mit} 16577 \mathrm{t}$ KAMPP, 1950, S. 44) und 1956 über 193 Einheiten, die mit einer Gesamttonnage von $25641 \mathrm{BRT}^{7}$ etwas über $80 \%$ des registrierten Schiffsbestandes ausmachten. Diese $Z$ ahl besagt außerdem, daß etwa $48 \%$ der im Besitz Dänemarks befindlichen Fischdampferflotte unter der Flagge der im dänischen Staatsverband relativ autonomen Färöer fahren. Wenn heute fast 5000 Personen ${ }^{8}$, d.h. etwa $32 \%$ aller färischen Erwerbstätigen, in der Fischerei beschäftigt sind, so kennzeichnet diese Tatsache die Bedeutung dieses Wirtschaftszweiges für den Arbeitsmarkt. $\mathrm{Zu}$ berücksichtigen ist allerdings, daß ein Teil der Seeleute sich auch auf ausländischen - vor allem isländischen - Schiffen anheuern läßt.

Während bis zu dem Anfang der zwanziger Jahre der Fang hauptsächlich im engeren Bereich der heimatlichen Inseln eine wesentliche Bedeutung besaß, sind seit diesem Zeitpunkt und mit zunehmender Größe der Schiffe auch die weiter entfernten Fischgründe erschlossen worden. Heute wird während der Fangsaison, hauptsächlich in der Zeit von Februar bis Mai, in den Gewässern rund um die Färöer oder südlich von Island gefischt; dagegen sucht man in der restlichen Zeit bis einschließlich September die Fanggebiete nördlich von Island und vor Grönland sowie um die BärenInsel und um Spitzbergen auf.

Vor allem werden Kabeljau und Hering gefangen, denen gegenüber die anderen Fische (Schellfisch, Heilbutt, Scholle u.a.) von geringerem Werte sind. 1956 belief sich die angelandete Menge an Fisch auf $116316 \mathrm{t}$, von denen allein $84708 \mathrm{t}$ auf $\mathrm{Ka}$ beljau und $23669 \mathrm{t}$ auf Hering entfielen. Während man den Kabeljau in zum Teil sehr modernen industriellen Betrieben zu Klippfisch verarbeitet und in zahlreiche Länder, vor allem aber nach südamerikanischen Staaten, nach Spanien und Italien, exportiert, wird der Hering, meistens als Salzfisch, von west- und mitteleuropäischen Staaten übernommen.

Eine gewisse Bedeutung hat auch der Walfang erreicht. Er wird von 5 Fangbooten aus betrieben, die ihre Beute nach der auf der Insel Streymoy befindlichen Kochereistation við Air bringen. Dort werden zur Zeit jährlich bis zu 200 Tiere zu Walöl, Walmehl usw. verarbeitet ${ }^{9}$.

Für die Bevölkerung von beträchtlichem wirtschaftlichen Nutzen ist ferner die Jagd auf Grindwale. Die bis zu $7 \mathrm{~m}$ langen und in Herden auftretenden Tiere werden von zahlreichen Booten aus auf den Strand getrieben und dort getötet. Bei einer einzigen Jagd erlegt man oft 200 Grindwale, deren Fleisch dann größtenteils eingesalzen wird (vgl. Abb. 2) ; die Gesamtbeute pro Jahr kann einige tausend Stück betragen.

Neben dem Fischfang ist die Landwirtschaft lebensnotwendig für die Bevölkerung der Färöer. Jahrhundertelang waren Ackerbau und Viehzucht, ergänzt durch Fischund Vogelfang, die Grundlagen der wirtschaftlichen Existenz, und diese Tatsache erklärt, daß die Färöer auch heute noch das Bild eines Bauernlandes aufweisen. Seit der Aufhebung des dänischen Handelsmonopols, d.h. seit etwa $100 \mathrm{Jahren}$, ist allerdings

6 Statistisk $\AA$ rbog, 1907.

7 Statistisk Årbog, 1957, S. 141. Im Register erfaßt sind nur Schiffe von mehr als 2o BRT.

8 Neuere statistische Angaben über die Färöer finden sich in dem Statistisk Årbog, 1957, S. $312-321$.

9 Die Anzahl der erlegten Wale schwankt beträchtlich; so betrug sie z.B. 1948: 275, 1950: 434, 1952: 52 (Betriebseinstellung der Station Lopra), 1953: 169, 1954: 31, 1955: 161, 1956 : 146. 
Abb. 2: Nach der Grindwaljagd werden die erlegten Tiere nach einem bestimmten Schlüssel an die einzelnen Familien der Stadt Tórshavn verteilt.

(Photo: OBERBECK 57/1321).

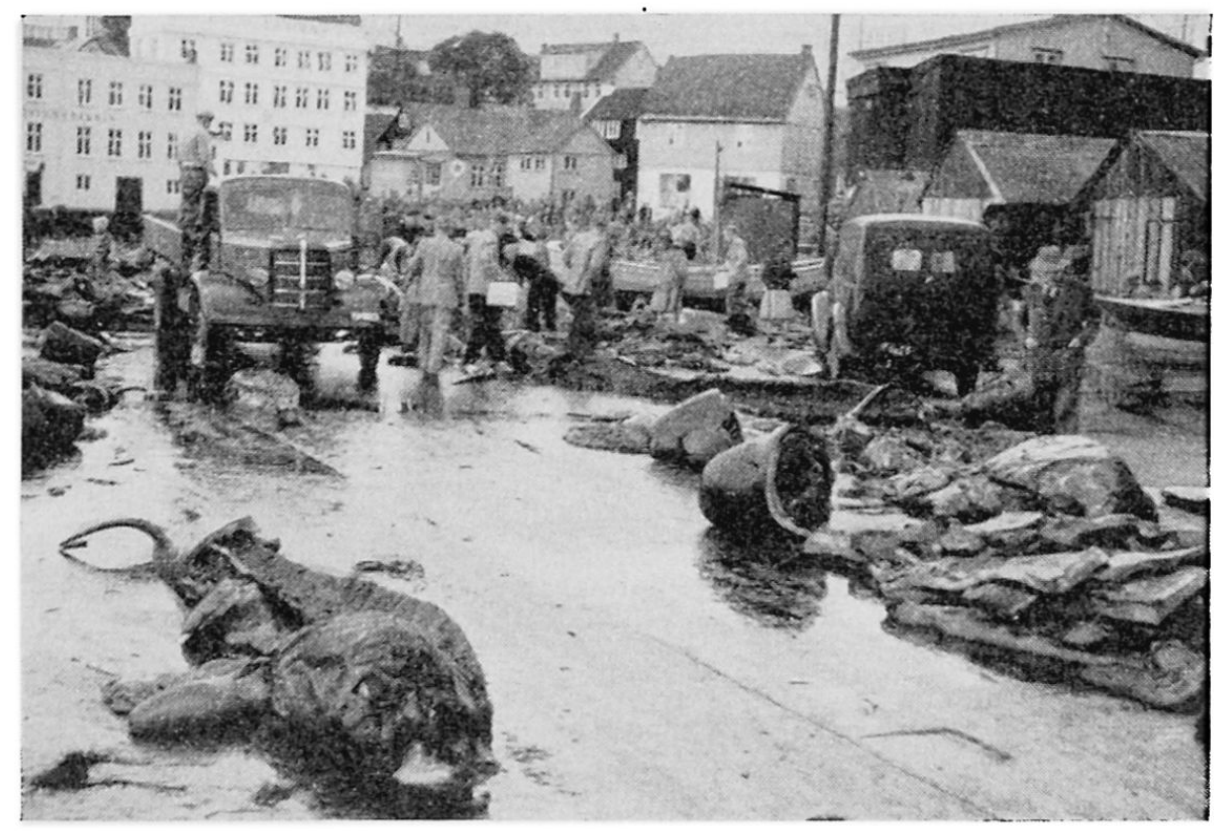

ein starkes Abwandern der bäuerlichen Bevölkerung in andere Wirtschaftszweige, vor allem in die Hochseefischerei, festzustellen. So lebten (nach KAMPP, 1950, S. 62) um 1800 noch $80 \%$ der Färinger (Gesamtzahl: 5265) von der Landwirtschaft, 1930 dagegen nur noch knapp 7\% der 24200 Bewohner ${ }^{10}$. Dieser Vorgang hat sich jedoch nicht dahingehend ausgewirkt, daß die agrarische Nutzungsfläche eingeschränkt wäre. Das Gegenteil ist vielmehr der Fall, und die «Indmark» (fär. böur.), wie das die Städte und Dörfer umgebende kultivierte Land bezeichnet wird, vergrößerte sich von 2,5\% der Gesamtfläche der Färöer im Jahre 1924 auf 3,1\% im Jahre 1930 und $3,7 \% 1935 ; 1947$ besaß es ungefähr die gleiche Ausdehnung (vgl. Oвerbeck, 1957, S. 189), 1957 jedoch sogar 4\% des Inselareals. Eine regionale Differenzierung ist insofern festzustellen als im Distrikt Suðuroy mit 6,5\% der höchste Anteil, bei den gebirgigeren Nordinseln jedoch mit 2,1\% der niedrigste erreicht wird; die übrigen Bezirke schwanken zwischen 5,5\% (Sandoy) und 2,6\% (Vágar) (vgl. Abb. 3).

Soweit die Indmark agrarisch genutzt wird, baut man in erster Linie Kartoffeln, Gerste, Rüben und Gemüse an. Vor allem aber dient das kultivierte Land der Gewinnung von $\mathrm{Heu}$ als Winterfutter für die Rinder, und zwar verwendet man es normalerweise im Wechsel 1 Jahr lang als Acker und 5 bis 6 Jahre als Grasland.

Der restliche und wesentlich größere Teil der Inseln wird als «Udmark» (fär. hagi) bezeichnet und lediglich als Viehweide genutzt. Besitzrechtlich interessant ist, daß die zum Teil sehr stark parzellierte Indmark ohne Flurzwang bewirtschaftet wird, die Udmark hingegen in Form von einzelnen «hagi» gemeinsames Eigentum von jeweils mehreren Bauern mit bestimmten Rechten hinsichtlich der Anzahl des zugelassenen Weideviehs ist. Es gibt 253 einzelne «hagi», von denen jedoch 18 nochmals untergliedert sind, so daß sich die Gesamtzahl auf 274 beläuft. $\mathrm{Zu}$ einer Ortschaft gehören - je nach der Größe des Dorfes - 1 bis 17 «hagi», und mehrere Siedlungen sind jeweils zu einer Gemeinde («Sogn») zusammengeschlossen.

Der färische Bauernstand weist eine bemerkenswerte Differenzierung auf, die einen beträchtlichen Einfluß auf die wirtschaftliche Situation ausübt. Es gibt einerseits den Eigentumsbauern (ódalsbóndi), der über ein freies Anwesen (ódal) verfügt. Infolge der üblichen Realteilung ist sein Indmarksbesitz jedoch derartig zersplittert, daß manche Höfe mit großen wirtschaftlichen Schwierigkeiten zu kämpfen haben. Hingegen hat der Königsbauer (kongsbóndi) sein Land (kongsjørd) pachtweise von der

10 Statistisk Årbog, 1954, S. 300. 


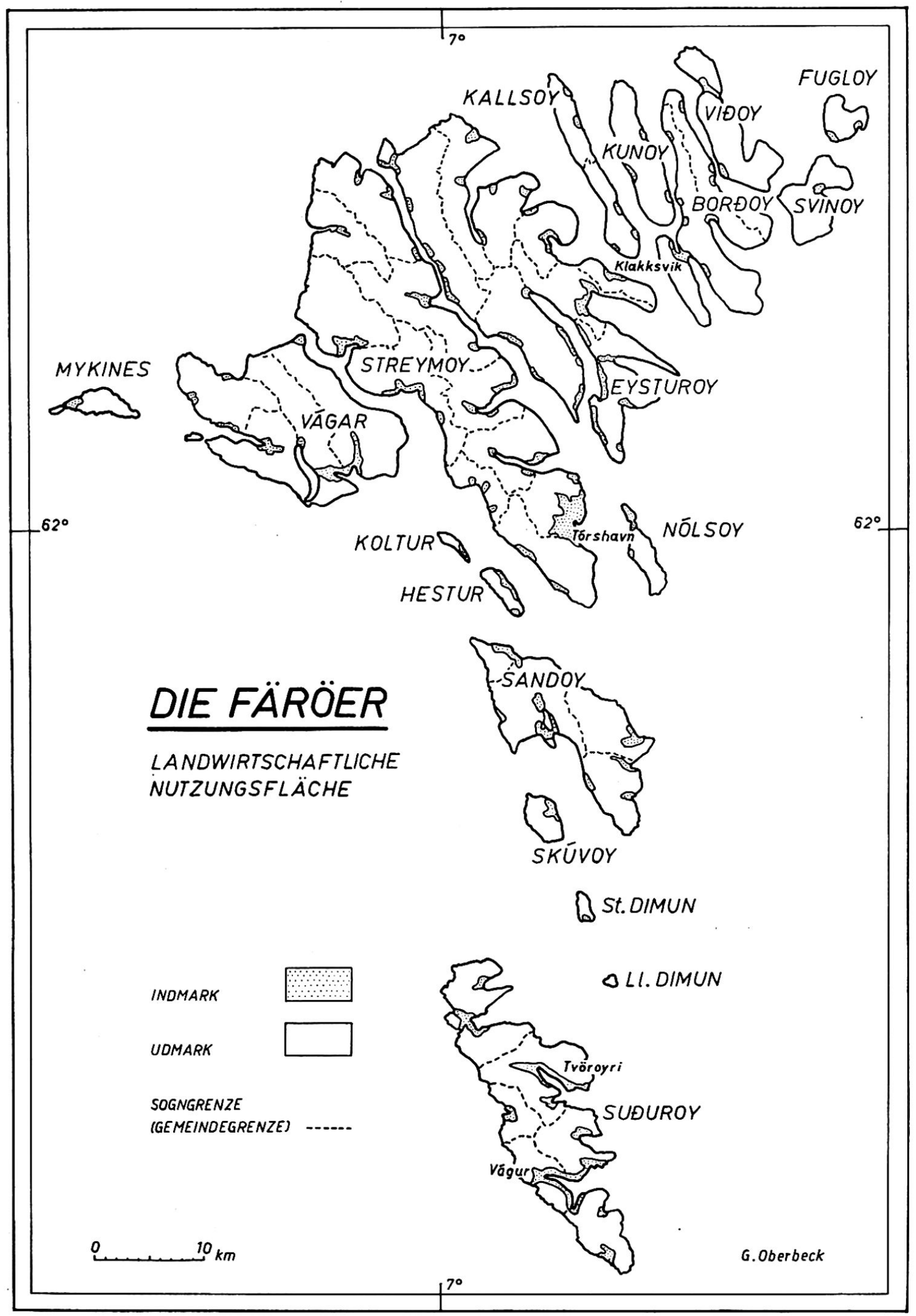

Abb. 3 
Abb. 4: Moderne

Fischtrocknungs-

und Verarbei-

tungsanlage in

Heygur (Strey-

moy). Im Vorder-

grund wird der

Klippfisch noch nach der alten Methode auf der Kaimauer und auf Gestellen getrocknet.

(Photo: OBERBECK 57/1308).

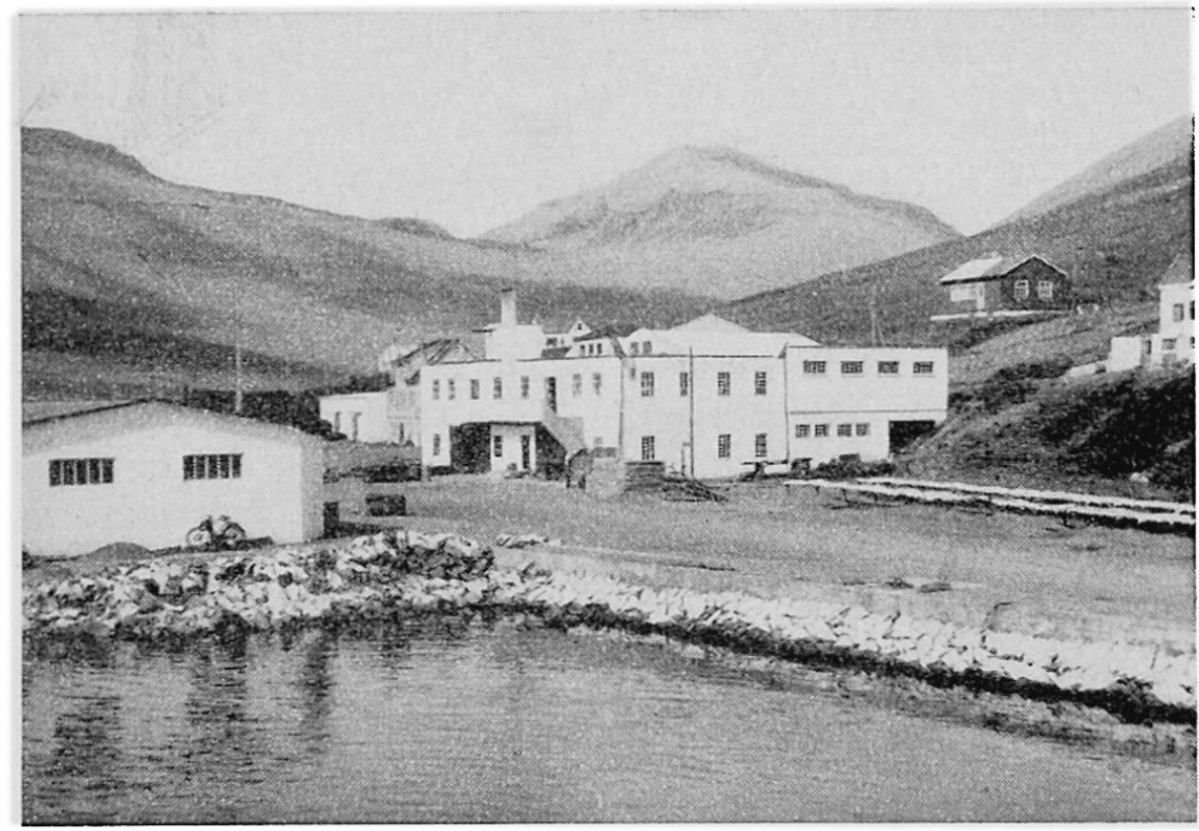

dänischen Krone erhalten, die das nach der Reformation 1538 beschlagnahmte Kirchengut auf diese Weise weiter nutzen ließ. Da nun bei dem Kongs-Bauern oder Staten-Fæster ${ }^{11}$ meistens der älteste Sohn den Hof übernimmt, also ein Erbpachtverhältnis vorliegt, wird das Land unverändert vererbt und eine Zerstückelung verhindert. Diese so unterschiedliche Entwicklung hat dazu geführt, daß aus rationellen Gründen in den Orten, in denen überwiegend Odals-Bauern wohnen, eine Verkopplung und Zusammenlegung (udskiftning) dringend nötig wurde. Sie ist in den Jahren 1927-28 begonnen und bisher für 13 Gemeinden abgeschlossen worden, zahlreiche weitere Siedlungen sollen aber noch folgen (vgl. OвеRвEсK, 1957, S. 195-98).

Für die Bauern der Färöer ist die Viehzucht und zwar vor allem die Schafhaltung von entscheidender Bedeutung. Nach den letzten statistischen Angaben ${ }^{12}$ belief sich der Schafbestand (1952) auf rund 63000 Tiere und 40000 Lämmer. Zu berücksichtigen ist jedoch, daß die Udmark nur einer bestimmten Anzahl von Tieren Weidemöglichkeit bietet und daher insbesondere im Winter sehr oft Futtermangel eintritt; dementsprechend werden zu Beginn des Oktobers zahlreiche Schafe, vor allem Jungtiere, geschlachtet, deren Fleisch getrocknet und als Winternahrung verwendet wird ${ }^{13}$. In den letzten Jahrzehnten hat auch die Haltung von Rindern zugenommen, deren Bestand zwischen 4300 und 4600 variiert; dagegen ist der Schweinebestand recht unwesentlich.

Von zahlreichen, meistens kleinen und abgelegenen Dörfern aus wird auch heute noch - wie seit Jahrhunderten - der Vogelfang betrieben. Aus den an den Steilküsten nistenden riesigen Schwärmen von Vögeln erlegen die Färinger unter oft lebensgefährlichen Umständen eine große Anzahl von Tieren, eine Art der Jagd, die zwar die Möglichkeit bietet, für den Winter zusätzlich Fleisch zu gewinnen, die jedoch für die gesamtwirtschaftliche Lage nur von geringer Wichtigkeit ist.

Stärkere Beachtung verdient die verarbeitende Industrie lediglich in den mit dem Fischfang zusammenhängenden $Z$ weigen. An zahlreichen Stellen der Inseln sind größere und kleinere Fabriken - zum Teil mit Mitteln aus dem Marshall-Plan - errichtet worden, die speziell den Kabeljau (Klippfisch!) oder den Hering verarbeiten (vgl.

11 Der Grundeigentümer ist heute der Staat, weshalb die Bezeichnung Staten-Fæster, d.h. Staats-Pachtbauer, eingeführt wurde.

12 Statistisk Årbog, 1954, S. 303.

131952 wurden annähernd 35000 Tiere geschlachtet. 
Abb. 4). Außerdem besteht eine bereits erwähnte, modern eingerichtete Walkocherei und -verarbeitungsanlage sowie eine Seifen- und Margarinefabrik, in der auch Walöl verwendet wird und die den Eigenbedarf der Inseln nahezu deckt. Die land- und viehwirtschaftlichen Produkte werden u.a. an 3 Molkereien und Käsefabriken, die ebenfalls hauptsächlich den färingischen Markt versorgen, geliefert. Die bei der Schafzucht anfallende Wolle - es handelt sich jährlich um $43000-46000 \mathrm{~kg}$ - wird größtenteils an eine Wollspinnerei in Tórshavn, die pro Jahr etwa $20000 \mathrm{~kg}$ abnimmt (1957), und an eine zweite in Klakksvik geliefert; daneben ist die Heimwirkerei noch ziemlich verbreitet. Entsprechend der Bedeutung der Schiffahrt für die Inseln verfügt Tórshavn über eine Werft, auf der sogar kleinere und mittlere Fischereifahrzeuge gebaut werden können, die aber - wie auch verschiedene andere kleinere Betriebe - in erster Linie Reparaturen ausführt.

Schwierig ist die Frage der Energieversorgung zu lösen. Die Färöer verfügen über ein Vorkommen von tertiärer Steinkohle, das jedoch lediglich auf Suðuroy abbauwürdig ist. Es handelt sich dabei um eine Kohle von nur geringem Heizwert (etwa 5000 Kalorien), die in zwei Flözen mit einer Mächtigkeit von etwa $1,50 \mathrm{~m}$ und $0,80 \mathrm{~m}$ ansteht (RAASchOU, 1937). Schon seit langem bekannt, hat man seit dem 17. Jahrhundert mehrmals versucht, diese Lager wirtschaftlich zu nutzen; infolge zu großer Schwierigkeiten wurden jedoch die wiederholt angelaufenen Unternehmungen immer wieder eingestellt (vgl. JohnstruP, 1873). Ein Wandel trat erst während des zweiten Weltkrieges ein, als die Färöer von England besetzt und von Dänemark abgeschnitten waren. 1940 wurde die Förderung in größerem Umfange aufgenommen, und zwar beträgt sie zur Zeit bei der Grube von Hvalböur etwa 12000 t und bei derjenigen von Trongisvágur etwa 3000 t pro Jahr ${ }^{14}$. Damit kann jedoch der Eigenbedarf der Inseln nicht gedeckt werden, und so ist man - vor allem in den abgelegenen Distrikten - immer noch auf die Verwendung von Torf, der an verschiedenen Stellen eine Stärke von 0,70-1,50 m erreicht, angewiesen.

Der Elektrizitätsversorgung dienen mehrere Kraftwerke in Vestmanna, Klakksvik, Vágur, Tvøroyri und Tórshavn, von denen das letztgenannte jedoch, das nur mit Dieselöl arbeitet, nach Fertigstellung des großen Wasserkraftwerkes in Vestmanna ${ }^{15}$

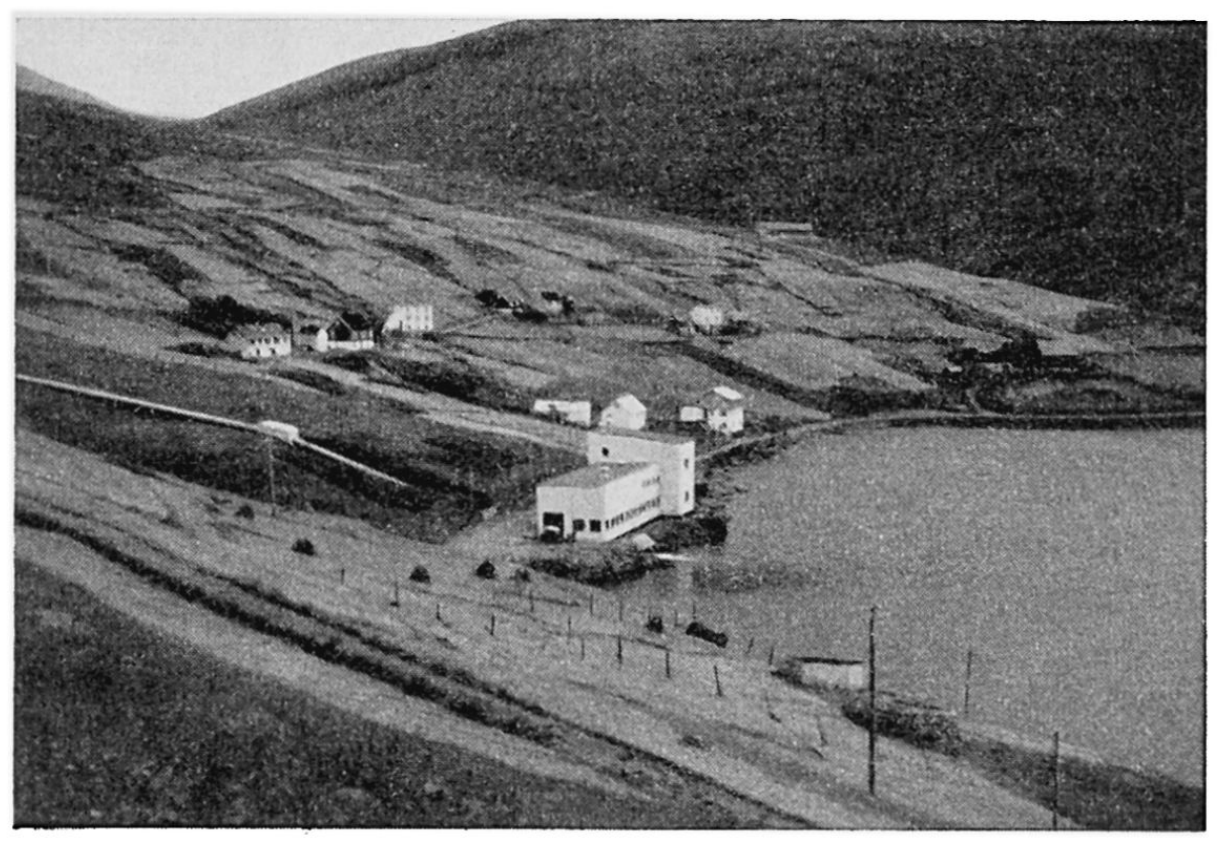

Abb. 5 : Modernes Elektrizitätswerk bei Vestmanna am Vestmannafjord (Streymoy). Im Vorder- und Hintergrund die den Ort umgebende Indmarksfläche.

(Photo: Oberbeck 57/1195).

14 Angaben des Grubendirektors in Hvalböur.

15 Es liefert maximal etwa 3,7 Mio Kwh./Jahr. 
(1954/55) (vgl. Abb. 5) lediglich noch Reservefunktionen hat. Von Vestmanna aus werden jetzt die Hauptinseln Streymoy und Eysturoy sowie Vágar und Teile von Borðoy mit elektrischem Kraftstrom beliefert.

Bei den zahlreichen Inseln mit ihren buchtenreichen Küsten ist der Schiffsverkehr lebensnotwendig, zumal manche Orte - selbst von nur wenige Kilometer entfernten Nachbarsiedlungen - infolge der gebirgigen Oberflächenbeschaffenheit auf dem Landwege gar nicht erreicht werden können. So stellen viele kleine Fracht- und Passagierschiffe nach genau festgelegten Fahrplänen die Verbindung zwischen den einzelnen Ortschaften her, und es ist durchaus möglich, bei einigermaßen günstigem Wetter wenn vielfach auch nur einmal in der Woche - zahlreiche der zum Teil sehr kleinen Dörfer zu erreichen. Welche Lokalbedeutung dieser Verkehr besitzt, zeigt sich darin, daß 1956/57 25 verschiedene Routen von 1 Dampfschiff (298 BRT) und 14 Motorschiffen (2-150 BRT) befahren wurden. Bei all diesen Verbindungen stellt sich heraus, daß Tórshavn als Verwaltungs- und Wirtschaftszentrum der Inseln auch verkehrsmäßig am besten bedient wird und für die meisten Linien den Anfangs- oder Endpunkt bildet (vgl. Abb. 7).

Die Landverbindungen sind infolge der starken Höhenunterschiede und des dementsprechenden Mangels an Straßen recht spärlich. Beispielsweise gibt es keine Insel, die man in ihrer gesamten Längserstreckung auf dem Landwege bereisen könnte. Auf den Nordinseln fehlen Verbindungsstraßen nahezu gänzlich. Lediglich Eysturoy, Streymoy, Vágar, Sandoy und Suðuroy verfügen über wenige Landstraßen (insgesamt etwa $300 \mathrm{~km}$ ), die dort allerdings die Schiffsverbindungen wirkungsvoll zu ergänzen vermögen; zahlreiche Last- und Personenwagen sowie Kleinbusse vermitteln den Verkehr zwischen den Hafenplätzen und den gar nicht oder an bestimmten Tagen nicht von den Schiffen angelaufenen Orten. Wie stark das Automobil trotz der wenigen Straßen bereits an Bedeutung gewonnen hat, sei an folgendem Beispiel verdeutlicht: 1938 gab es etwas über 100 Last- und Personenwagen; Ende $1956^{16}$ waren 323 Lastwagen und 201 Personenwagen bzw. Kleinomnibusse registriert, von denen wiederum 144 der gewerbsmäßigen Personenbeförderung dienten ${ }^{17}$.

Der Auslandverkehr wird von den Färöern aus durch eine einheimische Gesellschaft (Føroyar Skipafelagið) nach Kopenhagen und Lerwick (Shetlandsinseln) und durch eine dänische (Det Forenede Dampskipsselskab) regelmäßig nach Kopenhagen und Island durchgeführt; außerdem laufen isländische Schiffe auf der Route von Kopenhagen nach Reykjavik ebenfalls Tórshavn an. Hingegen fehlt eine Flugverbindung, da einerseits das Gelände für die Anlage eines größeren Flughafens sehr ungünstig, zum anderen zur Zeit das wirtschaftliche Bedürfnis nicht vorhanden ist. Zwar existiert noch aus der Zeit des zweiten Weltkrieges ein Flugfeld mit betonierter Piste auf der Insel Vágar, das jedoch für heutige Verkehrsflugzeuge zu klein ist und auch infolge des gebirgigen Geländes kaum erweitert werden kann. Wenn - womit in absehbarer Zeit zu rechnen ist - dennoch ein Flugplatz gebaut wird, so kommt dafür nur die an einigen Stellen etwas ebenere Insel Sandoy in Frage, bei Tórshavn selbst hingegen bietet sich keine Möglichkeit.

Der Export der Färöer beruht, wie bereits betont wurde, überwiegend auf der Ausfuhr von Fisch und Fischprodukten, und zwar belief sich diese im Jahr $1956^{\mathbf{1 8}}$ bei einem Gesamtexportertrag von etwa 93,1 Millionen Kronen allein auf 91,1 Millionen Kronen. An erster Stelle unter den einführenden Ländern stehen Spanien, die südamerikanischen Staaten Brasilien, Argentinien und Venezuela, das dänische Mutterland und Italien.

16 Statistisk Årbog, 1957, S. 316.

17 Hinzu kamen 147 Motorräder und -roller sowie 1 Kranken- und 1 Feuerwehrwagen.

18 Statistisk Årbog, 1957, S. 317-319. 


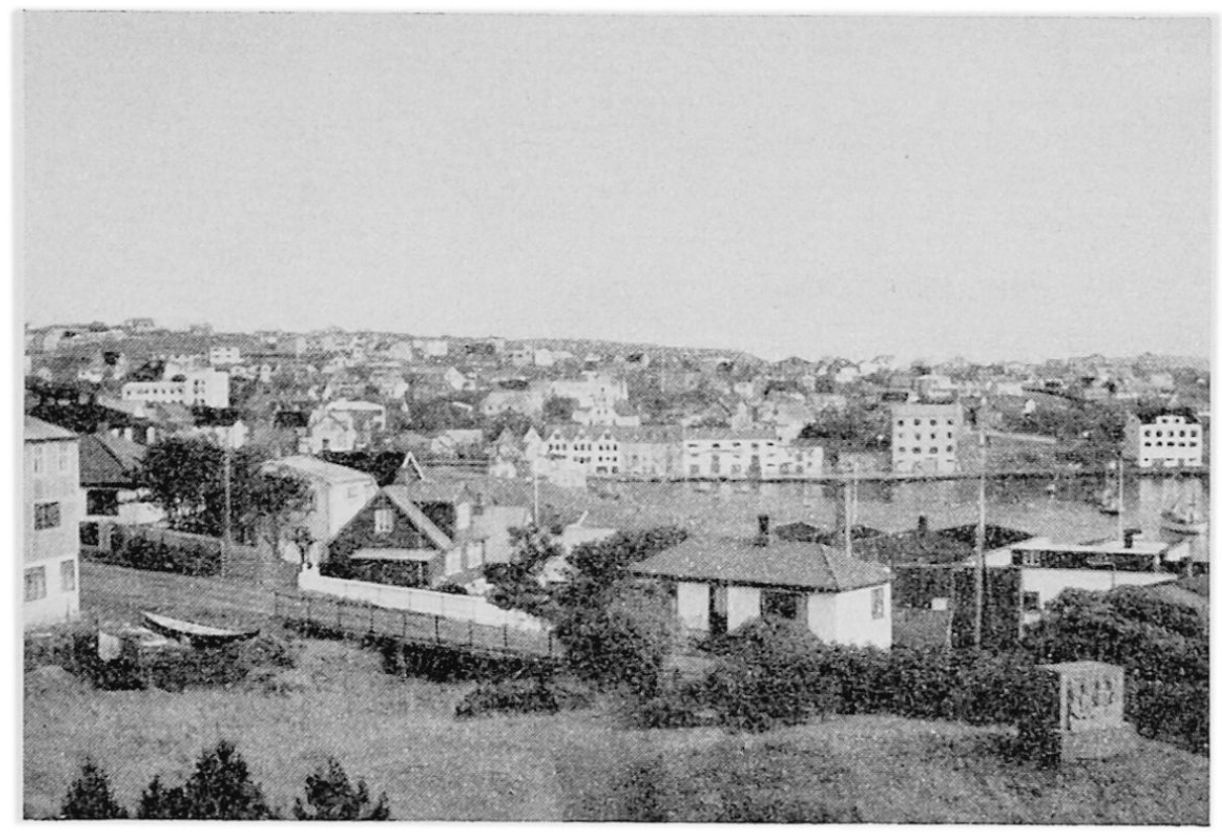

Abb. 6: Tórshavn, die Hauptstadt, ist das Kultur-, Verwaltungs-, Wirtschafts- und Verkehrszentrum der Inseln. Seine Einwohnerzahl stieg von $984(1880)$, auf $1303(1890)$, 2097 (1911), 3200 (1930), 5607 (1950) und beläuft sich seit $1955 / 56$ auf über 6100 .

(Photo: Oberbeck $57 / 1328)$.

In den meisten Jahren ist normalerweise mit einer durchaus ausgeglichenen ode positiven Zahlungsbilanz zu rechnen. Lediglich 1956 überstieg der Import um ein weniges die Ausfuhr, was zum Teil mit Exportüberschüssen aus den vorhergehenden Jahren zu erklären ist. Es fällt auf, daß bei den Einfuhrgütern Transportmittel, vor allem Kraftwagen, weit an erster Stelle stehen (15,5 Millionen Kronen), erst dann folgen Brennstoffe, Textilien, elektrische Artikel und schließlich Getreide. Die genannten Waren werden hauptsächlich aus Dänemark, aber auch aus Großbritannien und aus der Bundesrepublik Deutschland bezogen.

Eine zusammenfassende Betrachtung läßt erkennen, daß die wirtschaftliche Situation der Färöer durchaus günstig ist. Allerdings sind die Inseln beträchtlich von der Einfuhr zahlreicher lebensnotwendiger Güter abhängig, einer Einfuhr, die zu 95-98\% durch exportierte Fischprodukte gedeckt werden muß. So ist es verständlich, daß die Färinger den für die gesamte Bevölkerung so wichtigen Fischfang zu schützen versuchen, und in diesem Zusammenhang muß man auch - ausgelöst durch die isländischen Maßnahmen im Sommer 1958 - den Wunsch nach der Erweiterung der Hoheitsgewässer auf 12 Seemeilen sehen. Ferner wird angestrebt, den angelandeten Fisch möglichst im Lande weiter zu verarbeiten. Bestehende Fabriken sind zwar modernisiert und vergrößert worden, es bieten sich jedoch - besonders in der Fischkonservenindustrie - immer noch genügend Möglichkeiten der Erweiterung und damit der Wertsteigerung von Fischprodukten.

In der Landwirtschaft ist man bemüht, das Ackerland und die Indmark auszuweiten, um im Hinblick auf Agrarprodukte unabhängiger zu werden. Wenn dieses Ziel bisher nicht in dem gewünschten Umfang erreicht ist, so liegt das zum Teil daran, daß die Kongs-Bauern überwiegend infolge der relativ hohen Preise für landwirtschaftliche Erzeugnisse gut gestellt sind und wenig Initiative zur Kultivierung zeigen, daß es aber anderseits manchem Odals-Bauern an dem notwendigen Kapital und auch an Arbeitskräften mangelt. Für die Odals-Bauern ist im Zuge der weiteren Verkopplungen eine Verbesserung der besitzrechtlichen und damit auch der wirtschaftlichen Situation zu erwarten.

Eine Vergrößerung der Schafhaltung ist nicht wünschenswert und infolge der begrenzten Futterfläche auch nicht möglich; lediglich die Anzahl der Rinder würde bei einer Erweiterung der Wiesen in der Indmark noch etwas zunehmen können.

Die färische Industrie war - abgesehen von den fischverarbeitenden Betrieben bisher nur von geringer Bedeutung. Leider gingen eine Anzahl kleinerer Betriebe bald 


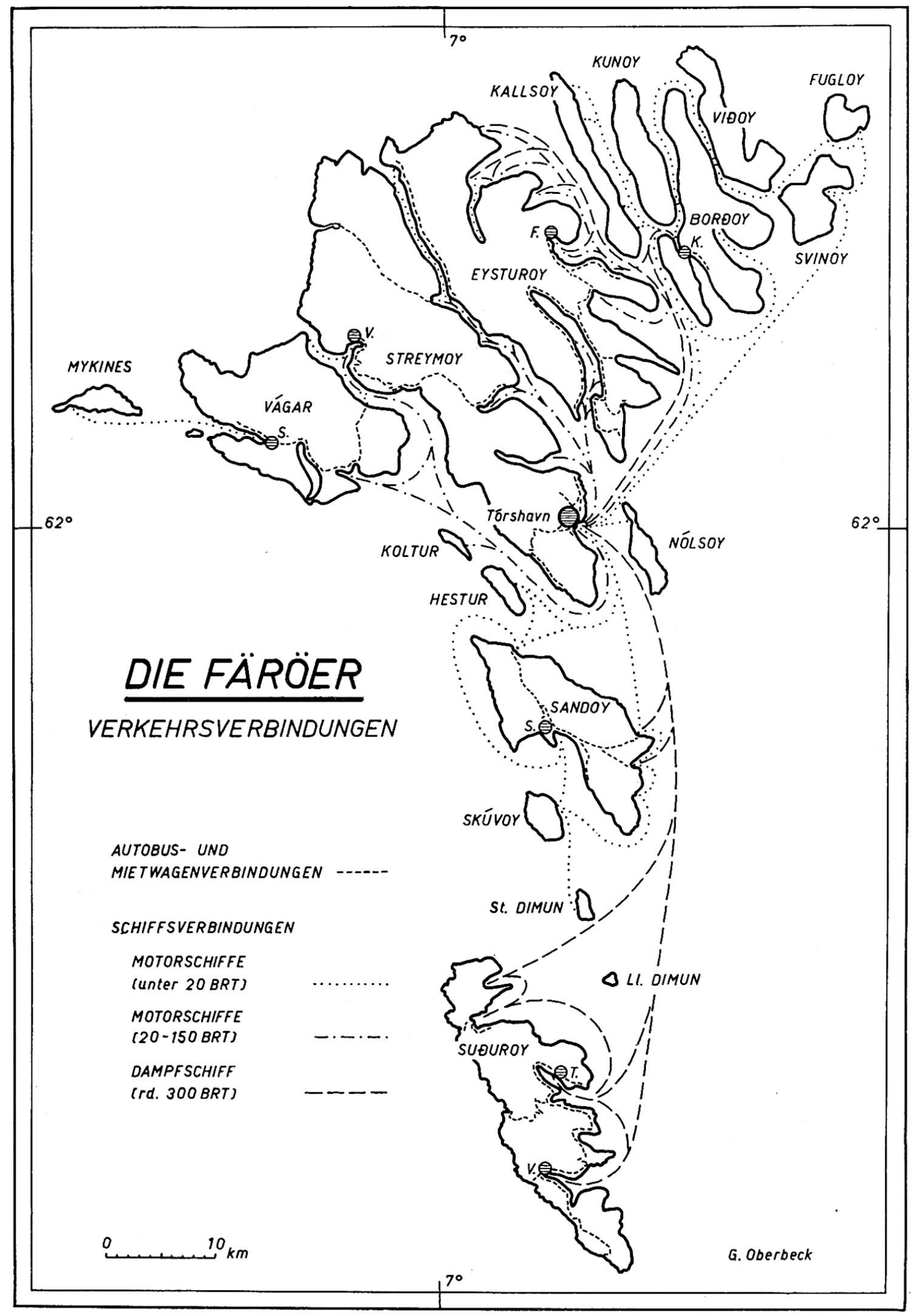

Abb. 7 
nach ihrer Gründung wieder ein. Es fehlte an Kapital, gelernten Arbeitskräften, modernen Maschinen und vielfach auch - bei 32400 Einwohnern - an dem nötigen inländischen Absatzmarkt. Eine etwas günstigere Position zeichnet sich für kleine und mittlere Betriebe im Hinblick auf die künftige Energieversorgung ab, da bei weiterer Ausnutzung der Wasserkräfte mit billigem Kraftstrom zu rechnen ist.

Die schwierige Verkehrslage auf den einzelnen Inseln versucht man durch den Straßenbau zu verbessern. Am günstigsten sind die Verhältnisse zur Zeit auf Suðuroy, wo man seit 1958 von Froðböur auf einer $40 \mathrm{~km}$ langen Straße bis nach Sunnböur gelangen kann. Notwendig wären weitere Verbindungen vor allem auf den Hauptinseln Streymoy und Eysturoy, auf denen bisher noch die gebirgige Oberfläche den technischen und finanziellen Möglichkeiten der Färinger eine Grenze gesetzt hat. Der lokale Schiffsverkehr genügt zwar nicht überall den Bedürfnissen, ist aber ausreichend. Die Verbindung mit Kopenhagen wurde 1958 verbessert, so daß man im Sommer nahezu in jeder Woche das Mutterland erreichen kann. Wünschenswert wenn auch wahrscheinlich nicht rentabel - wäre die Einrichtung einer Flugverbindung zum europäischen Festland, insbesondere nach Dänemark.

\title{
LITERATUR ÜB E RSICHT
}

BøGGILd, O. B.: Die Färöer. In: Übersicht über die Geologie von Dänemark, hrsg. von V. Madsen, S. 188-193. - Kopenhagen 1928. - Johnstrup, F.: Om Kullagene paa Færøerne. - København 1873. KampP, Aa. H.: Færøerne. - København 1950. - KrenN, E.: Föroyar und die Föroyinger. In: Mitt. d. Geogr. Ges. Wien, Bd. 86, 1943, H. 10-12, S. 329-351. - OBERBECK, G.: Landschaft und ländliche Siedlungen der Färöer. In: Jahrb. d. Geogr. Ges. zu Hannover f. d. Jahre 1956 und 1957, S. 179204. - Hannover 1957. - RaAschou, P. E.: Om Kulforekomsterne paa Færøerne. - København 1937.Rudolphi, H.: Die Färöer. In: Ztschr. d. Ges. f. Erdkd. zu Berlin, 1913, S. 162-193, S. 273-298. Trap, J. P.: Kongeriget Danmark, Færø Amt, 4. Udg. - København 1930. - Williamson, K.: The Atlantik Islands. - London 1948. - Færøerne, hrsg. v. d. Dansk-Færøsk Samfund, 2 Bde. København 1958. - Statistisk Årbog, hrsg. von dem Statistiske Department. - København 1907, 1954, 1957. - The Statesman's Year-Book 1958. - London 1958.

\section{DE LA SITUATION GÉOGRAPHIQUE ET ÉCONOMIQUE DES ILES FEROE}

Le groupe des îles Feroë comprenant 18 îles est de caractère très montagneux. Il est habité par une population d'environ 32000 habitants, dont la plus grande partie occupe les îles principales, Eysturoy, Streymoy et Suduroy. L'essentiel de la nourriture provient de la pêche, en particulier des morues et des harengs. Cette pêche constitue la principale activité des indigènes. En outre, on chasse la baleine et le globicéphale (Grind). L'agriculture joue un rôle important, elle n'est toutefois pratiquée que dans la région «Indmark». En revanche, la plus grande étendue du territoire sont des prairies où paissent des moutons et des bovins et des terrains non cultivés, «l'Udmark». L'industrie n'a quelque importance que dans l'exploitation des produits de la pêche. En matière de combustibles, ou trouve de la houille en petites quantités à Suduroy et de la tourbe. L'électricité est produite par la force hydraulique. Le trafic local est essentiellement maritime, car le réseau routier est encore rudimentaire. Les communications avec l'étranger se font par bateau, non par avion. Les importations et exportations du pays sont à peu près équilibrées. La situation économique générale des îles Feroë peut être considérée comme favorable.

\section{JAPANISCHE LANDNUTZUNGSMUSTER}

\author{
Hans Boesch
}

Schon früher sind vom Verfasser nordamerikanische Landnutzungsmuster beschrieben und deren Bedeutung im Rahmen landschaftskundlicher Untersuchungen dargestellt worden 1 . Im Folgenden seien einige charakteristische japanische Landnutzungsmuster erklärend beschrieben. Die folgende kurze Übersicht will außerdem referierend auf einige bei uns kaum bekannte

1 Вовsсн, H.: Amerikanische Landschaft - Neujahrsblatt Natf. Ges. Zürich 1955. 